\title{
Analysis of the Dose-Limited Spatial Resolution in Transmission Electron Microscopy
}

\author{
Eduardo Ortega ${ }^{1}$ and Niels de Jonge ${ }^{1,2}$
}

${ }^{1}$ INM - Leibniz Institute for New Materials, Saarbrücken, Saarland, Germany, ${ }^{2}$ Department of Physics, Saarland University, Saarbrücken, Saarland, Germany

Transmission electron microscopy (TEM) has played a canonical role to study the structure of materials and biochemical processes at the nanoscale. However, an unfortunate trade-off for the achievable resolution is the high electron dosage exerted into the specimen. High radiation dose has detrimental consequences for sensitive samples. Catalysts, zeolites, and many other macromolecules can only preserve their structures below a $100 \overline{\mathrm{e}} / \AA^{2}$ threshold [1]. In TEM, the spatial resolution is limited by the ratio of electrons available for detection, the energy spread of the electron source, and the broadening of the electron beam due to electron scattering. These components depend on the electron optics of the TEM instrument, the type of sample support, and the sample itself. Theoretical approaches to determine the dose-limited resolution are found elsewhere [2,3]. Our aim in this subject is to demonstrate the capabilities of those models to predict and comprehend the origins of resolution loss under standard TEM acquisition conditions.

The relation was systematically examined between the electron dose and the signal-to-noise ratio (SNR), chromatic aberration, and beam broadening. Our experimental setup consisted of a silicon microchip with a $50 \mathrm{~nm}$-thick electron transparent silicon nitride $(\mathrm{SiN})$ window. Both sides of the microchip contained a drop casted $2 \mu \mathrm{l}$ colloidal solution containing $15 \mathrm{~nm}$-diameter gold nanoparticles (AuNPs). This enabled us to observe the difference between the AuNPs at the electron entrance surface with their corresponding nanoparticles under the SiN window (Fig. 1A). To introduce a thickness gradient, imaging was conducted at different positions relative to the edge of the $\mathrm{SiN}$ window. As a substrate, the window doesn't produce diffraction contrast effects, which would have complicated our calculations, and it is the material of choice for liquid cell and in-situ TEM experiments. Images were acquired using a JEOL ARM 200F equipped with a cold field emission gun operated at $200 \mathrm{kV}$. A charge coupled device (CCD) camera (Gatan US1000XP) was used to record images. The pixel size was $0.94 \mathrm{~nm}$. The exposure time was chosen to control the electron dose $(\bar{e} D)$ from $10^{2}$ to $10^{-1} \overline{\mathrm{e}} / \AA^{2}$. As depicted in Fig. $1 \mathrm{~B}, \bar{e} D$ variations greatly changed the signal contrast. Images acquired with a frame acquisition of $1 \mathrm{~ms}$ reported a $\mathrm{SNR}<3$, making the nanoparticles untraceable. After data acquisition, line profiles of the AuNPs were extracted and adjusted to a Gaussian fit using a custom Matlab program (Fig 1C). Measurement of the spatial resolution was performed extracting the Gaussian 25-75\% rising edge width (r25-75) [4]. For this case, the r25-75 was used because the nanoparticles analyzed were larger than the resolution limit, making this parameter a more accurate measurement of "blurring".

Our experimental data show the changes in resolution for three distinctive cases. The first dominated by phase contrast (NP1), the second by beam broadening and bright field (BF) scattered contrast (NP2), and the third by a heavy chromatic aberration component (NP3). The graph in Fig. 1D displays the effect of electron dose when going under $5 \overline{\mathrm{e}} / \AA^{2}$. Below this range, the resolution scales with $\bar{e} D^{-1 / 4}$ as calculated in previous research work [3].

In summary, we analyzed the attainable resolution of dose-limited TEM taking into consideration thickness variations and diffraction contrast regime (phase contrast or BF amplitude contrast). The 
understanding of the upper and lower resolution limits of low-dose imaging will allow for an improved experimental design in TEM research [5].
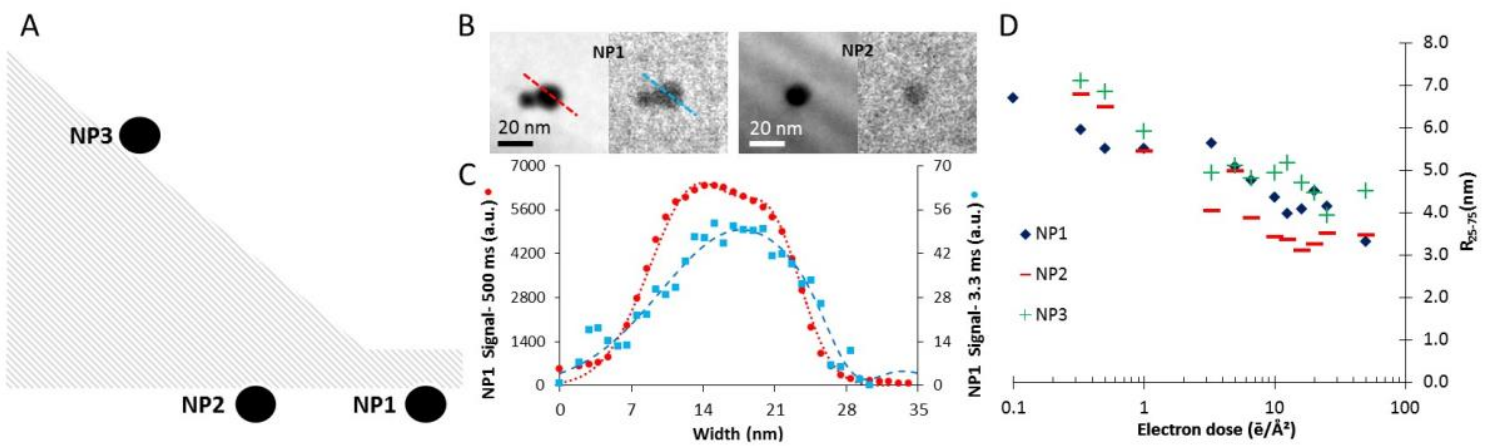

Figure 1. Analysis of the dose-limited spatial resolution of Transmission Electron Microscopy (TEM). (A) Schematically representation of a set of gold nanoparticles (AuNPs) used to measure the influence of the electron dose on the image contrast and resolution. (B) Experimental images of the AuNPs depicted as NP1 and NP2 in 'A'. The left image in each pair depicts the situation of an electron dose ( $\overline{\mathrm{e} D}$ ) of 50 $\overline{\mathrm{e}} / \AA 2$ while the right one an $\mathrm{e} \mathrm{D}=0.33 \mathrm{e} / \AA 2$. The poor contrast is expected as the SNR drops from 58.4 to 5.3. (C) Line profile over an AuNP including a Gaussian fit for the case of the NP1 illustrated in ' $\mathrm{B}$ '. Notice the change in detection intensity of 1/122 as acquisition time changed from 500 to $3.3 \mathrm{~ms}$. (D) Experimental measurement of the spatial resolution using the 25-75\% rising edge width (r25-75) as a measure of the obtained spatial resolution for the case of the AuNPs illustrated in 'A'.

\section{References}

[1] M Pan and PA Crozier, Ultramicroscopy 52 (1993) p. 487.

[2] L Reimer and H Kohl 2008 Transmission electron microscopy: physics of image formation (New York: Springer).

[3] N de Jonge, Ultramicroscopy 187 (2018) p. 113.

[4] N de Jonge et al. Ultramicroscopy 110 (2010) p. 1114.

[5] The authors thank E. Arzt for his support through INM. The research was supported by the German Research Foundation. 